\title{
THEORETICAL ANALYSIS OF PARTIAL-RENEWAL TYPE AIR FILTER*
}

\author{
NOBUTOSHI TANAKA**, KAZUTAKA MAKINO AND KOICHI IINOYA
}

Department of Chemical Engineering, Kyoto University, Kyoto, Japan

\begin{abstract}
A mathematical model of an air filter which is controlled by utilizing a pressure switch or a timer is formulated on the basis of some simple postulates. The dust-holding capacity, the renewal period, the fluctuation of pressure drop, the life time of a finite filter medium, etc., are numerically calculated for a wide range of practical conditions, and the merit of partial renewal, the comparison between pressure switch and timer types and the effect of length of medium on the performance of the air filter, etc., are discussed. These results are shown to be in good agreement with Higuchi's experimental data.
\end{abstract}

\section{Introduction}

A fibrous filter is one of the more commonly-used air filters, being particularly suitable for low dust concentrations. Its performance is hardly affected by the properties of dust or gas, and maintenance is easy because of its simple construction.

There are two types of air filter. One is a unit type, in which the filter medium is packed in a unit frame and replaced together with the frame after a certain period. The other is a long medium type, in which the medium is renewed by one of three medium renewal operations. These are entire-renewal, which is similar to the unit type; partial-renewal, in which the older portion of the medium in a filtering zone is replaced by the next fresh one; and continuousrenewal, in which the medium is continuously displaced at a specified velocity. Furthermore, two methods are used to control the renewal operation, utilizing a pressure switch or a timer respectively.

Higuchi et al. ${ }^{1)}$ have reported an interesting experimental result, which shows that a partial-renewal filter controlled by a pressure switch can hold about twice the dust load collected by the entire-renewal or the unit-type filters. However, the theoretical analysis of the partial-renewal operation has not yet been given completely ${ }^{2}$. Therefore, an analytical model rigorously formulated on the basis of some simple postulates is presented here, and the dust-holding capacity, the renewal period, the fluctuation of pressure drop, etc., are numerically calculated for a wide range of practical conditions and compared with the experimental results ${ }^{1)}$.

* Received on April 19, 1972

Presented at Okayama meeting of the Soc. of Chem. Engrs., Japan, Okayama, July 11, 1972

** Kyoto Prefectural Institute of Public Health

\section{Formulation of the Partial Renewal Model}

As shown in Fig. 1(a), let us suppose that the filtering zone may be divided into $k$ equal parts, and that the medium may be intermittently displaced by $L / k$ at every renewal. Therefore, both dust loads $m_{i}$ and filtering velocities $u_{i}$ show step-wise distributions. Then the partial renewal model may be formulated by using the following postulates.

Postulate 1 The pressure drop across the medium $\Delta p$ can be expressed in terms of $m$ and $u$ as follows ${ }^{3}$.

$$
\Delta p=u^{n}\left(a+b m^{q}\right)
$$

where $a, b, n$ and $q$ are constant values which are experimentally obtained and depend on the properties of both medium and dust.

Postulate $2 \Delta p$ should not exceed $\Delta p_{f}$, which is a maximum pressure drop.

Postulate 3 The average filtering velocity $\bar{u}$ is always to be kept constant, independently of $\Delta p$.

Postulate 4 The product of dust concentration $c$ and collection efficiency $\eta$ is constant.

These postulates seem reasonable, but postulate 3 will be discussed further later. Postulate 4 is necessary to deal theoretically with an air filter and to obtain many of the conclusions mentioned below. The concentration $c$ may be regarded as constant as an engineering approximation, but $\eta$ might not always be constant for the usual filter media. However, Higuchi et al. ${ }^{1)}$ have reported that $\eta$ is approximately constant over the whole filtering period for a commercial air filter.

The following circumstances prevail during partial renewal operation. Each dust load $m_{i}$ increases with filtering time $t$, causing an increase in the pressure drop $\Delta p$. However, the filtering velocities $u_{i}$ increase in some sections and decrease in others in accordance with postulate 3 . After a while, the me- 


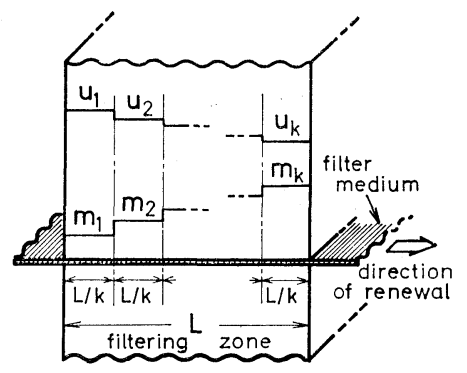

(a) step-wise distribution of $u_{i}$ and $m_{i}$

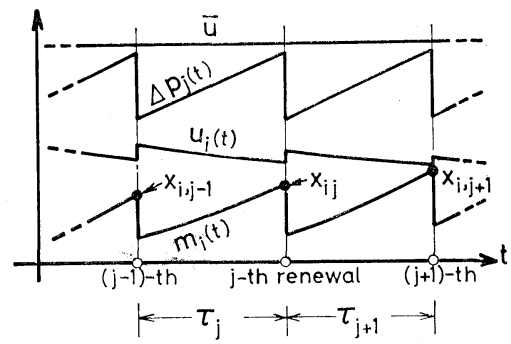

(b) $m_{i}, u_{i}, \Delta p$ and $\bar{u}$ vs. $t$
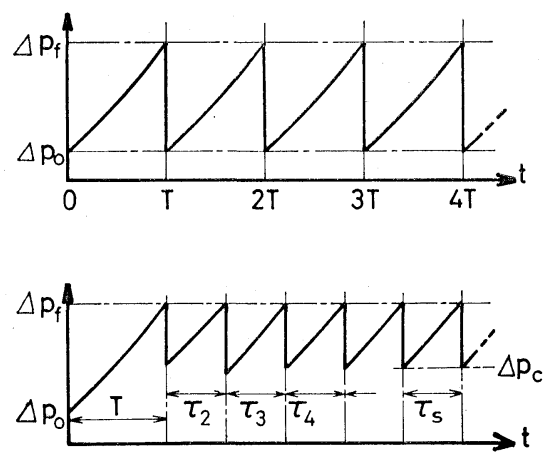

(c) $\Delta p$ vs. $t$ in a complete renewal

Fig. 1 Outline of medium renewal of air filter

dium is displaced as the specified renewal condition is attained at $t=\tau$. Then the pressure drop, dust loads, and filtering velocities change suddenly. That is, the relationships of $\Delta p, m_{i}$ and $u_{i}$ versus $t$ are saw-toothed curves, as shown in Fig. 1(b).

The mathematical equations for these procedures are as follows.

$$
\begin{gathered}
\frac{d m_{i}(t)}{d t}=c \eta u_{i}(t) \\
u_{i}(t)=\left[\frac{\Delta p(t)}{a+b\left\{m_{i}(t)\right\}^{q}}\right]^{1 / n} \\
\vec{u}=\frac{1}{k} \sum_{i=1}^{k} u_{i}(t)
\end{gathered}
$$

where $i=1,2, \cdots, k$ and $0 \leqq t \leqq \tau$.

When $k=1$, that is for entire renewal, the pressureswitch type is identical to the timer type, as shown in Fig. 1(c), and the dust load on the medium discharged at every renewal is constant. By using Eqs. (2), (3) and (4) under the limiting condition that the medium is not renewed before $\Delta p$ attains $\Delta p_{f}$, the solutions of the entire renewal situation are given as follows.

$$
\begin{aligned}
& M=c \eta \bar{u} T=\left(\frac{\Delta p_{f}-\Delta p_{0}}{b \bar{u}^{n}}\right)^{1 / q} \\
& \Delta p_{f}=\bar{u}^{n}\left(a+b M^{q}\right) \\
& \Delta p_{0}=\bar{u}^{n} a
\end{aligned}
$$

The above values are used as references for defining the appropriate dimensionless quantities, and Eqs.(2), (3) and (4) are transformed as follows.

$$
\begin{gathered}
\frac{d \tilde{m}_{i}(\tilde{t})}{d \tilde{t}}=\tilde{u}_{i}(\tilde{t}) \\
\tilde{u}_{i}(\tilde{t})=\left[\frac{\Delta \widetilde{p}(\tilde{t})}{\alpha+(1-\alpha)\left\{\tilde{m}_{i}(\tilde{t})\right\}^{q}}\right]^{1 / n} \\
k=\sum_{i=1}^{k} \tilde{u}_{i}(\tilde{t})
\end{gathered}
$$

where $\alpha$ is defined by

$$
\alpha \equiv \frac{\Delta p_{0}}{\Delta p_{f}}
$$

$i=1,2, \cdots k$, and $0 \leqq \tilde{t} \leqq \tilde{\tau}$.

Only initial and final values of the solutions of Eqs. (8), (9) and (10) are discussed here because the amount of discharged dust is the important factor. As shown in Fig. 1(b), let $\tilde{x}_{i j}$ denote the dimensionless values of the dust load in the filtering zone just before the $j$-th renewal, that is

$$
\tilde{x}_{i j} \equiv \tilde{m}_{i}\left(\tilde{\tau}_{j}\right) \quad(i=1,2, \cdots, k)
$$

Then, because every renewal discharges a unit increment of the medium, the values of dust load just after the $(j-1)$-the renewal can be expressed as follows.

$$
\begin{aligned}
& \tilde{m}_{1}(0)=0 \\
& \tilde{m}_{i}(0)=\tilde{x}_{i-1}, j-1 \quad(i=2, \cdots, k)
\end{aligned}
$$

Therefore, by the use of Eq.(9), the integration of Eqs. (8) and (10) from 0 to $\tilde{\tau}_{j}$ respectively gives

$$
\begin{aligned}
y\left(\tilde{x}_{1 j}\right) & =y\left(\tilde{x}_{2 j}\right)-y\left(\tilde{x}_{1, j-1}\right) \\
\vdots & =y\left(\tilde{x}_{k j}\right)-y\left(\tilde{x}_{k-1, j-1}\right)=\int_{0}^{\tau_{j}}\{\Delta \widetilde{p}(\tilde{t})\}^{1 / n} d \tilde{t} \\
k \tilde{\tau}_{j} & =\sum_{i=1}^{k} \tilde{x}_{i j}-\sum_{i=1}^{k-1} \tilde{x}_{i, j-1}
\end{aligned}
$$

where

$$
y(\tilde{x}) \equiv \int_{0}^{x}\left\{\alpha+(1-\alpha) \tilde{x}^{q}\right\}^{1 / n} d \tilde{x}
$$

By substituting Eq.(9) into Eq.(10), the dimensionless initial and final pressure drops are obtained as follows.

$$
\begin{aligned}
\Delta \widetilde{p}_{j}(0) & =\left[\frac{k}{\alpha^{-1 / n}+\sum_{i=1}^{k-1}\left\{\alpha+(1-\alpha)\left(\tilde{x}_{i, j-1}\right)^{q}\right\}^{-1 / n}}\right]^{n} \\
\Delta \widetilde{p}_{j}\left(\tilde{\tau}_{j}\right) & =\left[\frac{k}{\sum_{i=1}^{k}\left\{\alpha+(1-\alpha)\left(\tilde{x}_{i j}\right)^{q}\right\}^{-1 / n}}\right]^{n}
\end{aligned}
$$

The following initial conditions are held at the beginning of operation because the medium has no dust load.

$$
\tilde{x}_{1,0}=\tilde{x}_{2,0}=\cdots=\tilde{x}_{k, 0}=0 \quad \text { (I.C.) }
$$

Finally, the renewal conditions are given as follows.

$$
\begin{aligned}
\Delta \widetilde{p}_{j}\left(\tilde{\tau}_{j}\right) & =1 & & \text { (pressure-switch type) } \\
\tilde{\tau}_{j} & =\tilde{\tau}_{c} & & \text { (timer type) }
\end{aligned}
$$

In summary, the partial-renewal model (pressureswitch type) consists of the recurrence Eqs. (14), (18) and (20) with the initial condition Eq.(19), and $\tilde{\tau}_{j}$ is 
Table 1 Values used for computation

$k=2,5,10$ and 20

$\alpha=0.02$ to 0.5

$\left.\left.\left.\left.\left.\begin{array}{lll}n=1.6 \\ q=1.5\end{array}\right\} \quad \begin{array}{ll}2.0 \\ 2.0\end{array}\right\} \quad \begin{array}{l}2.0 \\ 0.7\end{array}\right\} \quad \begin{array}{l}1.0 \\ 2.0\end{array}\right\} \quad \begin{array}{l}1.0 \\ 0.7\end{array}\right\}$

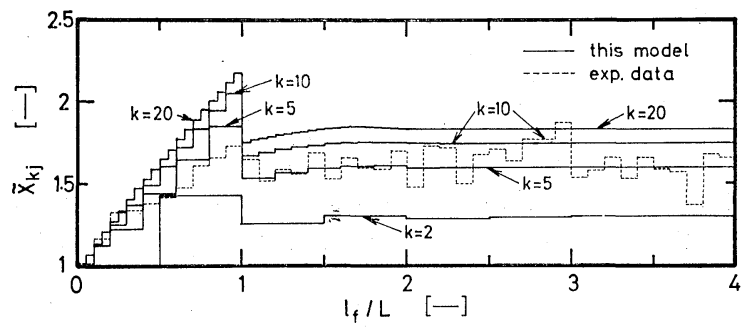

Fig. 2 Start-up values for discharged dust load (dimensionless) at $n=1.6, q=1.5$, and $\alpha=0.394$

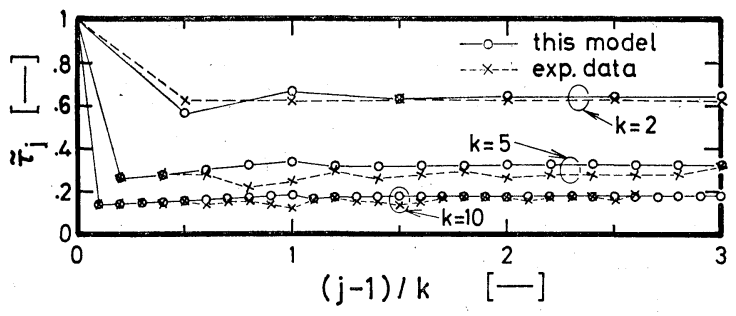

Fig. 3 Start-up values for renewal period (dimensionless) at $n=1.6, q=1.5$, and $\alpha=0.394$

calculated by substituting the above solutions into Eq.(15). Stated more precisely, $\tilde{x}_{2 j}, \cdots, \tilde{x}_{k j}$ are calculated from Eqs.(14) and (16), after assuming a value of $\tilde{x}_{1 j}$. Then, these values are substituted into Eq.(18). If $\Delta p\left(\tilde{\tau}_{j}\right)=1$, the correct solutions are obtained. If not, the above calculations are repeated using an iterating process. On the other hand, the timer type model consists of Eqs.(14), (15) and (21) with Eq.(19). $\Delta \widetilde{p}_{j}\left(\tilde{\tau}_{c}\right)$ is calculated by substituting the above solutions into Eq.(18), and should not exceed unity because of postulate 2 .

In particular, when both $\tilde{x}_{i j}$ and $\tilde{\tau}_{j}$ attain their steady-state values $\tilde{x}_{i s}$ and $\tilde{\tau}_{s}$, respectively, Eq.(15) yields the mass balance equation

$$
k \tilde{\tau}_{s}=\tilde{x}_{k s}
$$

This model, which will be shown later to be very useful for the design and operation of an air filter, has four significant parameters, namely, $k, \alpha, n$ and $q$. $k$ and $\alpha$ may be selected by the designer, however $n$ and $q$ are dependent on the properties of the dust and the medium.

Variable $\tilde{x}_{k j}$ is a measure of the renewal operation from the economic standpoint, because it represents the dust load on the medium discharged at every renewal.

\section{Results of Calculation on Pressure-Switch Type}

Using the partial-renewal model, computation is

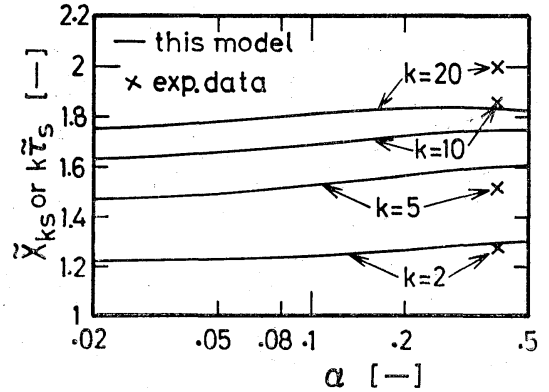

Fig. 4 Relationship between the steady-state discharged dust load (dimensionless), or the steady-state renewal period (dimensionless), and parameter $\alpha$ at $n=1.6$ and $q=1.5$

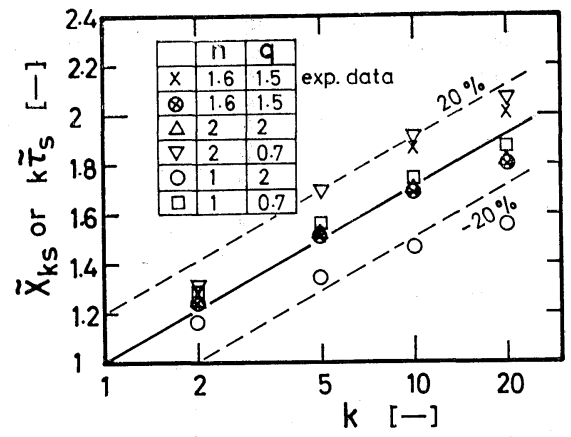

Fig. 5 Relationship between discharged dust load (dimensionless), or renewal period (dimensioless), and renewal ratio in the steady state

carried out from the initial condition without a dust load defined by Eq.(19), until $\tilde{x}_{i j}$ reaches the final steady-state value. The values of the parameters used for computation are listed in Table 1. These values cover all possible ranges of the properties of both medium and dust, and all operating conditions for an air filter.

\subsection{Start-up values of $\tilde{\boldsymbol{x}}_{k j}$ and $\tilde{\boldsymbol{\tau}}_{j}$}

In Figs. 2 and 3, theoretical examples of start-up values of the dust load discharged at every renewal $\left(\tilde{x}_{k j}\right)$ and renewal period $\left(\tilde{\tau}_{j}\right)$ are given for the same conditions as the experimental results. Although the values of $\tilde{\tau}_{j}$ are discrete, they are tied with lines in Fig. 3 to make their tendencies clear.

Both figures show that, independently of $k, \tilde{x}_{k j}$ and $\tilde{\tau}_{j}$ reach their steady-state values after a renewal length of about $L$ and after one renewal, respectively. In other words, partial renewal can be condisered as a steady-state operation in an engineering sense. This conclusion holds for all possible values of the parameters.

Therefore, the renewal period can be approximated as follows.

$$
\left.\begin{array}{l}
\tilde{\tau}_{1}=1 \\
\tilde{\tau}_{j} \fallingdotseq \tilde{\tau}_{s} \quad(j=2,3, \cdots)
\end{array}\right\}
$$

That is, except for the first renewal, the pressureswitch type becomes the same as the timer type, in which the medium is renewed at constant periods. Therefore, for constant average filtering velocity and

VOL. 5 NO. $4 \quad 1972$ 


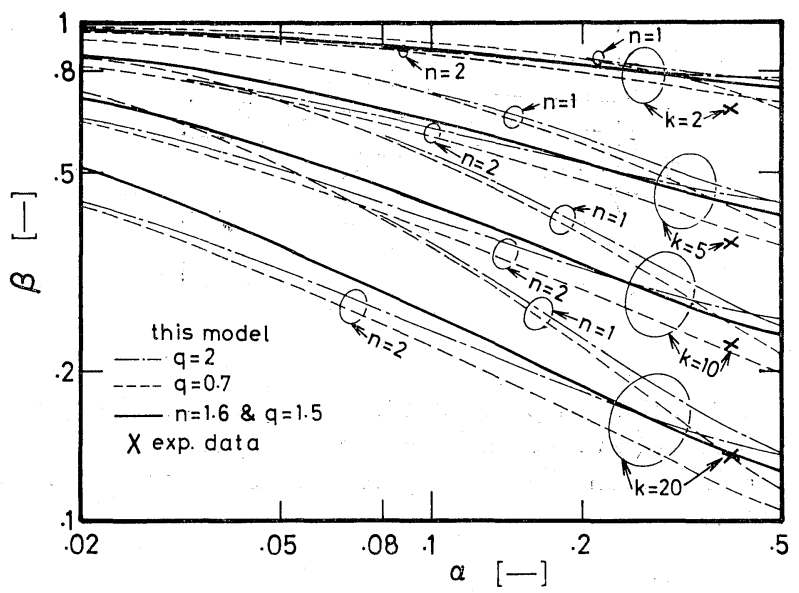

Fig. 6 Effect of parameters $\alpha, k, n$ and $q$ on fluctuation of pressure drop at steady state

dust concentration (postulates 3 and 4) the timer type is more desirable than the pressure-switch type in practice, because the timer is cheaper and more reliable than the pressure switch.

\subsection{Effect of $\boldsymbol{\alpha}$ on $\tilde{\boldsymbol{x}}_{k s}$ or $\tilde{\boldsymbol{\tau}}_{s}$}

The relationships of $\tilde{x}_{k s}$ or $\tilde{\tau}_{s}$ and $\alpha$ given in Fig. 4 show that $\alpha$ has little effect on $\tilde{x}_{k s}$ and $\tilde{\tau}_{s}$. This tendency holds for the whole range of values of $n$ and $q$. In other words, even if a powerful fan is used to increase $\Delta p_{f}$, or if some other kind of medium is used to decrease $\Delta p_{0}$, the merit of the partial-renewal operation would scarcely be affected.

\subsection{Effect of $\boldsymbol{n}$ and $\boldsymbol{q}$ on $\tilde{\boldsymbol{x}}_{k s}$ or $\tilde{\boldsymbol{\tau}}_{s}$}

Using $\tilde{x}_{k s}$ and $\tilde{\tau}_{s}$ at $\alpha=0.1$ as typical values, the relationship of $\tilde{x}_{k s}$ or $\tilde{\tau}_{s}$ versus $k$ is shown in Fig. 5 for various combinations of $n$ and $q$. Unexpectedly, it is concluded that $\tilde{x}_{k s}$ increases to about 2 with increasing value of $k$. That is, the dust load on the modium discharged at every partial renewal becomes about twice as much as that of every replacement when operating in the entire renewal mode. Further, even if $n$ and $q$ are unknown, the following equations can be used within about $20 \%$ error.

$$
\tilde{x}_{k s}=k \tilde{\tau}_{s}=1+0.7 \log k
$$

In other words, each partial renewal length should be as short as possible, and for given values of $k, x_{k s}$ and $\tau_{s}$ can be estimated by Eq.(24) as far as $M$ and $T$ are known.

\subsection{Fluctuation of pressure drop}

The curves of $\Delta p$ versus $t$ are saw-toothed in form, as shown in Fig. 1(d). However, according to the conclusion of Section 3.1, only the steady-state value is calculated here. By using Eqs.(17), (18) and (20), $\Delta p_{c}$ is given as follows.

$$
\Delta \widetilde{p}_{c} \equiv \frac{\Delta p_{c}}{\Delta p_{f}}=\left[\frac{k}{k+\alpha^{-1 / n}-\left\{\alpha+(1-\alpha)\left(\widetilde{x}_{k s}\right)^{q}\right\}^{-1 / n}}\right]^{n}
$$

Let us introduce the dimensionless parameter $\beta$ defined as

$$
\beta \equiv \frac{\Delta p_{f}-\Delta p_{c}}{\Delta p_{f}-\Delta p_{0}}=\frac{1-\Delta \tilde{p}_{c}}{1-\alpha}
$$

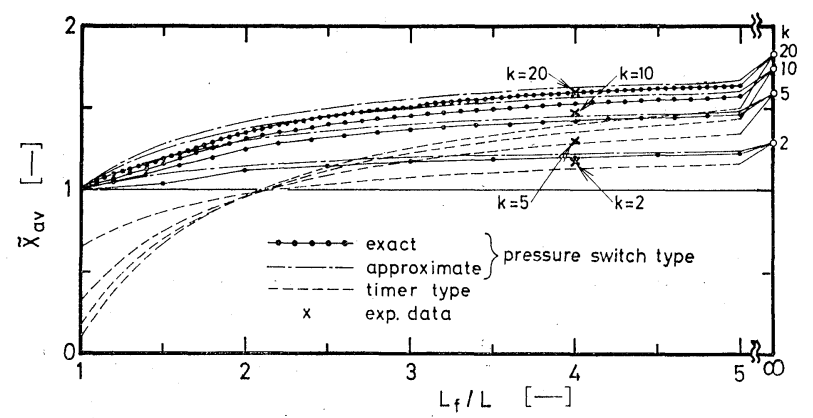

Fig. 7 Relationship between dust holding capacity and length of finite medium at $n=1.6, q=1.5$, and $\alpha=0.349$

where $\beta=1$ means the maximum change with time of pressure drop (entire renewal), and $\beta=0$ means no change, (partial renewal where $k$ tends to infinity).

The computed values of $\beta$ are plotted in Fig. 6, from which $\Delta p_{c}$ can be estimated for given values of parameters. It is concluded that $\beta$ is strongly dependent on change of $k$ and $\alpha$, moderately on that of $n$, and hardly on that of $q$. The conclusion reached in the previous section, that the partial renewal length should be as short as possible, can also be obtained from the standpoint of the minimum change of pressure drop.

\subsection{Discussion of timer type}

It is clear that the renewal period of the timer type, $\tau_{c}$, should be taken to equal $\tau_{s}$, which is the steadystate renewal period of the pressure-switch type. Also, $\Delta p\left(\tau_{c}\right)$ should be equal to $\Delta p_{f}$ to discharge as much dust per unit length of the medium as possible, and $\Delta p$ may hardly exceed $\Delta p_{f}$, even if $\tau_{c}$ is taken to be equal to $\tau_{s}$. For the design of the partial-renewal system (the timer type), $\tau_{c}$ can be easily estimated by Eq.(24) for a given value of $k$, provided that $T$ is known.

3.6. Dust-holding capacity and lifetime of a finite medium

Based on the above conclusions, the dust-holding capacity, which is denoted by the average dust load, $\tilde{x}_{a v}$ and the life time $\theta$ are as follows.

$$
\begin{gathered}
\tilde{x}_{a v}=\frac{L}{k L_{f}}\left(\sum_{j=1}^{h-1} \tilde{x}_{k j}+\sum_{i=1}^{k} \tilde{x}_{i h}\right) \\
\theta=T \sum_{j=1}^{h} \tilde{\tau}_{j}
\end{gathered}
$$

where

$$
h=1+\frac{\left(L_{f}-L\right) k}{L}
$$

$\tilde{x}_{a v}$ and $\theta$ are two measures of economic operation for a finite medium, and $\tilde{x}_{a v}$ approaches $\tilde{x}_{k s}$ asymptotically as $L_{f}$ becomes longer. The first term of Eq. (27) indicates the total weight of dust discharged by $(h-1)$ renewals, and the second term the total weight of the dust in the filtering zone just before the $h$-th renewal.

By using Eqs.(15) and (23), the above equations can be expressed as follows. 


$$
\begin{aligned}
& \left\{\begin{array}{c}
\tilde{x}_{a v} \fallingdotseq \frac{k\left\{1+(h-1) \tilde{\tau}_{s}\right\}}{k+h-1} \\
\quad(\text { pressure-switch type) } \\
\theta \fallingdotseq\left\{1+(h-1) \tilde{\tau}_{s}\right\} T
\end{array}\right. \\
& \left\{\begin{array}{c}
\tilde{x}_{a v}=\frac{k h \tilde{\tau}_{s}}{k+h-1} \\
\theta=h \tilde{\tau}_{s} T \quad \text { (time type) }
\end{array}\right.
\end{aligned}
$$

As an example, the relationship between $\tilde{x}_{a v}$ and $L_{f}$ is shown in Fig. 7, in which the approximate values obtained from Eq.(30) are compared with the exact values calculated from Eq.(27). $\quad \tilde{x}_{a v}$ and $\theta$ can be estimated for given $L_{f}$, if $k$ and $T$ are selected in advance.

From the above equations, it is concluded that a longer medium is more economical in practice than a shorter one. For the pressure-switch type it is necessary that the length of the medium be about three to five times as long as $L$ so that $\widetilde{x}_{a v}$ attains $90 \%$ of the maximum value $\tilde{x}_{k s}$. On the other hand, for the timer type the length must be about ten times $L$. Therefore, the pressure-switch type is more desirable than the timer type when the length of medium must be restricted.

\subsection{Discussion on the validity of this model}

The validity of this model can be examined by reference to the experimental data which were obtained at conditions corresponding to the values $k=2$ to 20 , $n=1.6, q=1.5$ and $\alpha=0.394$. The comparisons between the values calculated from the model and the experimental results are shown in Figs. 2 to 7 .

Inspection shows that the calculated values are in good agreement with the experimental data. Therefore, it seems reasonable to conclude that the model represents adequately the operation mechanism of the partial-renewal type air filter. However, further experimental studies with various combinations of $\Delta p_{0}$ and $\Delta p_{f}$ should be conducted.

\subsection{Effect of change of the average filtering velocity on} performance

Postulate 3 does not always hold in actual operation of an air filter. However, the dust loads, such as $\tilde{x}_{i j}$, $\tilde{x}_{k s}$ and $\tilde{x}_{a v}$ for changing average velocity, are the same as those for constant average velocity, if $\alpha$ is replaced by $\alpha^{\prime}$. This replacement is not substantial because of the small effect of $\alpha$ on $\tilde{x}_{k s}$ and so on. On the other hand the renewal periods, such as $T^{\prime}$, $\tau_{s}^{\prime}$ and the pressure drop, such as $\Delta p_{c}^{\prime}$, for changing average velocity, are different from those for constant average velocity. That is, $\tau_{j}^{\prime}$ cannot be taken as the time interval necessary to attain both a specified pressure drop and dust load. Therefore, the following approximate equations are proposed.

$$
\begin{aligned}
& T^{\prime} \fallingdotseq \frac{2 \bar{u}_{f} T}{\bar{u}_{0}+\bar{u}_{f}} \\
& \tau_{s}^{\prime} \fallingdotseq \frac{2 \bar{u}_{f} \tilde{\tau}_{s}}{\bar{u}_{c}+\bar{u}_{f}}
\end{aligned}
$$

Also, $\Delta p_{c}^{\prime}$ may be modified as follows:

$$
\Delta \widetilde{p}_{c}^{\prime}=\Delta \widetilde{p}_{c}\left(\frac{\bar{u}_{c}}{\bar{u}_{f}}\right)^{n}
$$

In Eqs. (35) and (36), it is necessary to replace $\alpha$ by $\alpha^{\prime}$. It is noted that the constant average velocity $\bar{u}$ should be equal to $\bar{u}_{f}$, and $\Delta p_{0}$ and $\Delta p_{f}$ are expressed as

$$
\begin{aligned}
& \Delta p_{0}=a \bar{u}_{0}^{n} \\
& \Delta p_{f}=\bar{u}_{f}^{n}\left(a+b M^{q}\right)
\end{aligned}
$$

Therefore, most of the results and conclusions for constant average velocity mentioned in the previous sections are applicable, provided the above modifications are reasonably acceptable.

\section{Conclusion}

A partial-renewal model is formulated using the solutions for entire-renewal as a reference subject to a few reasonable postulates. For wide practical ranges of parameters, the model gives many important results and conclusions, and its validity is established by comparison with experimental data.

The main conclusions obtained in this study are as follows :

i) Partial renewal is remarkable superior to entire renewal from the economical standpoints of medium consumption and smaller fluctuation of pressure drop and flow rate.

ii) The merits of partial renewal are little affected by changes in the properties of medium and dust, or by maximum pressure drop.

iii) More economical operation would be obtained by use of a timer working to fixed time schedule (Eq. (23)) instead of a pressure switch for conditions of constant flow rate and dust concentration.

iv) The renewal period for the timer type can be estimated easily from Eq.(24) for a given renewal ratio, when the period of an entire renewal is known.

v) The dust-holding capacity and lifetime of a finite length medium can be estimated easily by Eqs.(30) to (33), and a longer medium is more economical in practice than a shorter one.

vi) The pressure-switch type is superior to the timer type when the medium is restricted in length.

vii) The fluctuation of pressure drop can be roughly estimated by use of Fig. 6.

viii) Even if the average filtering velocity changes with pressure drop, the conclusions obtained for constant average velocity can be applied by introducing simple modifications.

\section{Appendix \\ Exercise \\ When a filter is operated as either an entire-renewal type or a $20 \%$ partial-renewal one $(k=5)$, how long can the medium be used in each case, assuming the following conditions?

$$
\begin{aligned}
& a=5 \mathrm{~kg} / \mathrm{m}^{2}(\mathrm{~m} / \mathrm{sec})^{n}, \quad b=15 \mathrm{~kg} / \mathrm{m}^{2} /(\mathrm{m} / \mathrm{sec})^{n}\left(\mathrm{~kg} / \mathrm{m}^{2}\right)^{q} \\
& c=10^{-6} \mathrm{~kg} / \mathrm{m}^{3}, \quad L=1 \mathrm{~m}, \quad L_{f}=10 \mathrm{~m} \\
& n=1.0, \quad q=1.5, \quad \quad \bar{u}=1 \mathrm{~m} / \mathrm{sec}
\end{aligned}
$$$$
\Delta p_{f}=20 \mathrm{mmH}_{2} \mathrm{O}, \quad \eta=0.9
$$

Furthermore, how large is the fluctuation of the pressure drops in each case if a pressure switch is used to control the renewal operation?

Answer

The dust load $M$ is calculated from Eq.(5) as follows. 


$$
M=\left(\frac{20-5}{15 \times 1}\right)^{1 / 1.5}=1 \quad\left[\mathrm{~kg} / \mathrm{m}^{2}\right]
$$

Then

$$
T=\frac{M}{c \cdot \eta \cdot \bar{u}}=\frac{1}{10^{-6} \times 0.9 \times 1} \doteqdot 1.11 \times 10^{6} \quad[\mathrm{sec}]
$$

i.e., life time of an entire-renewal type

$=T \frac{L_{f}}{L}=1.11 \times 10^{6} \times \frac{10}{1}=1.11 \times 10^{7}[\mathrm{sec}]=128$ [days]

Now consider the $20 \%$ partial-renewal type. From Eq. (29)

$$
h=1+\frac{(10-1) \times 5}{1}=46
$$

and from Eq.(24)

$$
\tilde{\tau}_{s}=\frac{1+0.7 \log 5}{5}=0.296
$$

By substituting Eqs.(iii), (iv) and (v) into Eq.(31), the following lifetime is obtained.

Life time of the $20 \%$ partial-renewal type

$$
\begin{aligned}
& =1.6 \times 10^{7} \quad \text { [sec] } \\
& =185[\text { days] }
\end{aligned}
$$$$
=(1+45 \times 0.296) \times 1.11 \times 10^{6}
$$

Comparison of these two results indicates the superior economy of the partial-renewal type of opertation.

The above conditions give $\alpha=\frac{5}{20}=0.25$. Therefore, from Fig. 6 , it is seen that $\beta=1$ for $k=1$ (entire renewal) and $\beta=0.54$ for $k=5$ (20\% partial renewal).

Then, from Eq.(26)

$$
\begin{aligned}
\Delta p_{c}= & 20-1 \times(20-5)=5 \quad\left[\mathrm{mmH}_{2} \mathrm{O}\right] \\
& (\text { for entire renewal) } \\
\Delta p_{c}= & 20-0.54 \times(20-5)=12 \quad\left[\mathrm{mmH}_{2} \mathrm{O}\right] \\
& (\text { for } 20 \% \text { partial renewal })
\end{aligned}
$$

Thus the pressure drop fluctuations for operation in the partial-renewal type are over twice the magnitude of those for entire-renewal operation.

\section{Nomenclature}

$a, b, n, q=$ constants used in Eq.(1)

$c \quad=$ dust concentration in air

$=$ dummy index defined by Eq. (29)

$=$ renewal ratio, that is ratio of length of filtering zone to renewal length

$L \quad=$ length of filtering zone

$L_{f} \quad=$ length of medium

$l_{f} \quad=$ length of discharged medium

$[-]$

= dust load on medium discharged at every entire renewal

$\left[\mathrm{kg} / \mathrm{m}^{2}\right]$ $m(t) \quad=$ dust load on medium $\quad\left[\mathrm{kg} / \mathrm{m}^{2}\right]$

$\Delta p(t) \quad=$ pressure drop across medium $\quad\left[\mathrm{Kg} / \mathrm{m}^{2}\right]$

$\Delta p_{c} \quad=$ pressure drop just after renewal at steady state $\quad\left[\mathrm{Kg} / \mathrm{m}^{2}\right]$

$\Delta p_{f} \quad=$ maximum pressure drop $\quad\left[\mathrm{Kg} / \mathrm{m}^{2}\right]$

$\Delta p_{0} \quad=$ pressure drop across medium without dust $\left[\mathrm{Kg} / \mathrm{m}^{2}\right]$

$T \quad=$ period of entire renewal [sec]

$t \quad=$ filtering time $\quad[\mathrm{sec}]$

$u(t) \quad=$ filtering velocity $\quad[\mathrm{m} / \mathrm{sec}]$

$\bar{u} \quad=$ average filtering velocity, see Eq.(4) $\quad[\mathrm{m} / \mathrm{sec}]$

$\bar{u}_{0}, \bar{u}_{c}, \bar{u}_{f}=$ average filtering velocities corresponding to $\Delta p_{0}, \Delta p_{c}$ and $\Delta p_{f}$, respectively $\quad[\mathrm{m} / \mathrm{sec}]$

$x_{a v} \quad=$ average dust load on finite length of medium, see Eq. (27) $\quad\left[\mathrm{kg} / \mathrm{m}^{2}\right]$

$x_{i j} \quad=$ dust load on the $i$-th part of medium in filtering zone just before the $j$-th renewal $\quad\left[\mathrm{kg} / \mathrm{m}^{2}\right]$

$=$ function of $x$, defined by Eq.(16)

= parameter defined by Eq.(11)

$=$ parameter $\equiv \alpha\left(\bar{u}_{f} / \bar{u}_{0}\right)^{n}$

$=$ parameter difined by Eq.(26)

= life time of a finite medium

$=$ renewal period

$=$ renewal period of the timer type

$=$ collection efficiency

$\eta \quad=$ collection

$$
\begin{array}{ll}
\langle\tilde{m} & =m / M \\
\tilde{x} & =x / M \\
\tilde{u} & =u / \bar{u} \\
\Delta \tilde{p} & =\Delta p / \Delta p_{f} \\
\tilde{t} & =t / T \\
\tau & =\tau / T, \text { etc. }
\end{array}
$$

$\langle$ superscripts〉

$$
\sim \quad=\text { dimensionless values as shown above }
$$

$i \quad=$ order in filtering zone, $=1,2, \ldots k$

$j \quad=$ number of renewals

$s \quad=$ steady state

\section{Literature Cited}

1) Higuchi, T. and A. Iba: J. Res. Assoc. Powder Tech., 6, $317(1969)$

2) Iinoya, K., K. Makino and Y. Fukui: Reports of the Asahi Glass Foundation for the Contribution to Industrial Technology, 18, 35 (1971)

3) Iinoya, K.: "Shujin Sochi (Shinpan)", p.181', Nikkan Kogyo Shinbun, Japan (1970) 